\title{
A Case of Holoprosencephaly with Atypical CT Findings and No Facial Anomaly
}

\author{
EIICHIRO HONDA, TAKASHI HAYASHI, KAZUTO SHOJIMA*, \\ KAZUHIKO MORITAKA*, HOTETSU SHIMAMOTO* \\ AND TAKAO SHOJIMA* \\ Department of Neurosurgery, St. Mary's Hospital and *Department of Neurosurgery,
Kurume University School of Medicine, Kurume, 830 Japan
}

Received for publication May 10, 1985

\begin{abstract}
Summary: We report a case of a 3 month old female infant, presenting with an increased tension of the fontanelle, cramp and irritability. No abnormality was noted during the periods of her mother's pregnancy and delivery. Although the CT findings in this case apparently revealed seemingly the pattern of severe subdural effusion, the cerebral ventricle was proved to be single. Cerebral angiography showed the anterior cerebral artery running along the frontal base. The sylvian triangle was not formed in the arterial phase. In the venous phase, deep cerebral veins (straight sinus, internal cerebral and the Galen veins) were not visualized although a variety of abnormal vein net works were observed. Holoprosencephaly was diagnosed this time. In this case, the histological findings at the operation confirmed that the subdural effusion-like space on CT was actually the dorsal sac. As DeMyer mentioned, this case was compatible with the morphology of holoprosencephaly in that cerebral parenchyma was retroflexed in a posterior direction. The patient did not show any facial malformations and the CT findings were also atypical appearance. The definite diagnosis was, therefore, made possible only by cerebral angiography in this case.
\end{abstract}

Key words : holoprosencephaly - cerebral angiography - subdural effusion dorsal sac - retroflexion.

\section{Introduction}

Holoprosencephaly is generally thought to be an anomaly due to a growth disturbance in the process of differentiation of the prosencephalon in the 5 th week of the embryonic stage. The diagnosis of this disease only by CT scan is extremely diffidult except in typical cases.

There are cases of holoprosencephaly which are misdiagnosed as extensive subdural effusion on CT scan, being without facial anomaly as was experienced in this case. In this case report, we report a case of atypical holoprosencephaly, with special reference to its pathogenesis.

\section{Cases Reports}

Patient: A 3-month-old female

Chief complaints: Increased tension of the anterior fontanelle, convulsions and irritability. Pregnancy and delivery were

Reprint Requests to: Eiichiro Honda, M.D. Department of Neurosurgery, St. Mary's Hospital. Tsubuku Honmachi, Kurume, 830 Japan. 
normal.

Body weight at birth-2980g; height- 47 $\mathrm{cm}$, circumferences of the head and the chest $31.4 \mathrm{~cm}$ and $32 \mathrm{~cm}$ respectively.

Present illness: An inceased tension of the fontanelle was found on July 9, 1979, one month after birth. From July 17, irritability and hyperreaction to sound appeared and fever and vomiting developed. She was brought to the Department of Pediatrics of the University on the following day, and transferred to our clinic because of the finding of an abnormal subdural space on $\mathrm{CT}$ scan.

Findings on admission: The patient was found to be asthenic and weak in suckling and crying. Neurological examination showed that the lower limbs were spastic and the tendon reflexes were increased. Neither a Moro reflex nor rigidity of the neck was observed. Slight conjugate deviation to the left was noted. Although she had a mild coryza with stridor, her fever was thought to be due to dehydration.

Neuroradiological findings: CT scan revealed what seemed be the pattern of extensive subdural effusion in the bifrontal area. The cerebral ventricle was found to be single.

The posterior fossa was normal (Fig. 1). Metrizamide was not transferred to the giant subdural-like space by CT cisternography. Although a non-symmetrical and irregular subarachnoid space was observed in the supratentorial parenchyma, the subarachnoid space in the posterior fossa was found to be normal (Fig. 2).

In carotid angiography by the Selginger method, the anterior cerebral artery was found to run along the frontal base. The azygous anterior cerebral artery was not seen on angiography, because the anteriorposterior view was not demonstrated due to sudden bradycardia. The right anterior cerebral artery was not developed. The bilateral sylvian triangles were not present (Fig. 3).

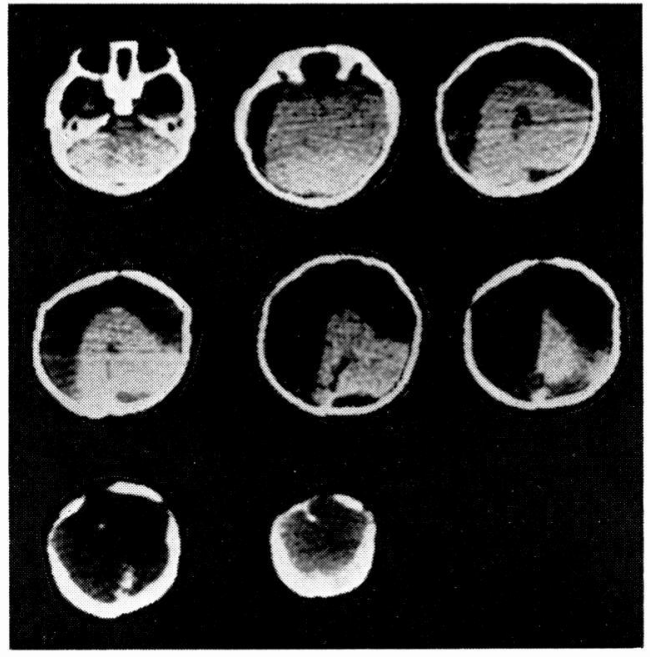

Fig. 1. Subdural effusion was suspected on CT scan. The supraventricular system was monoventricular. An unknown low density area was observed at the posterior of the skull.

In the venous phase, the structure of the deep cerebral veins (straight sinus, internal cerebral vein) were not demonstrated while only various draining veins were noted. In addition, the sagittal sinus was not visualized (Fig. 4). No abnormalities in the vertebrobasilar artery were noted.

Left fronto-temporal craniotomy was performed to determine the cause of the sequestrated fluid in the subdural-like space and to reduce it. Degenerated membranous tissues existed below the dura mater.

On removing these tissues, xanthocromic fluid appeared. In the internal space, a rudimentary falx cerebri was noted in the frontal base. A part of the cerebral parenchyma and the falx cerebri were connected with fascicular tissuse (Fig. 5). The membranous tissues below the dura mater were identified histologically to be the cerebral parenchyma. The surface of the inner most layer was covered with subependymal cells of the 


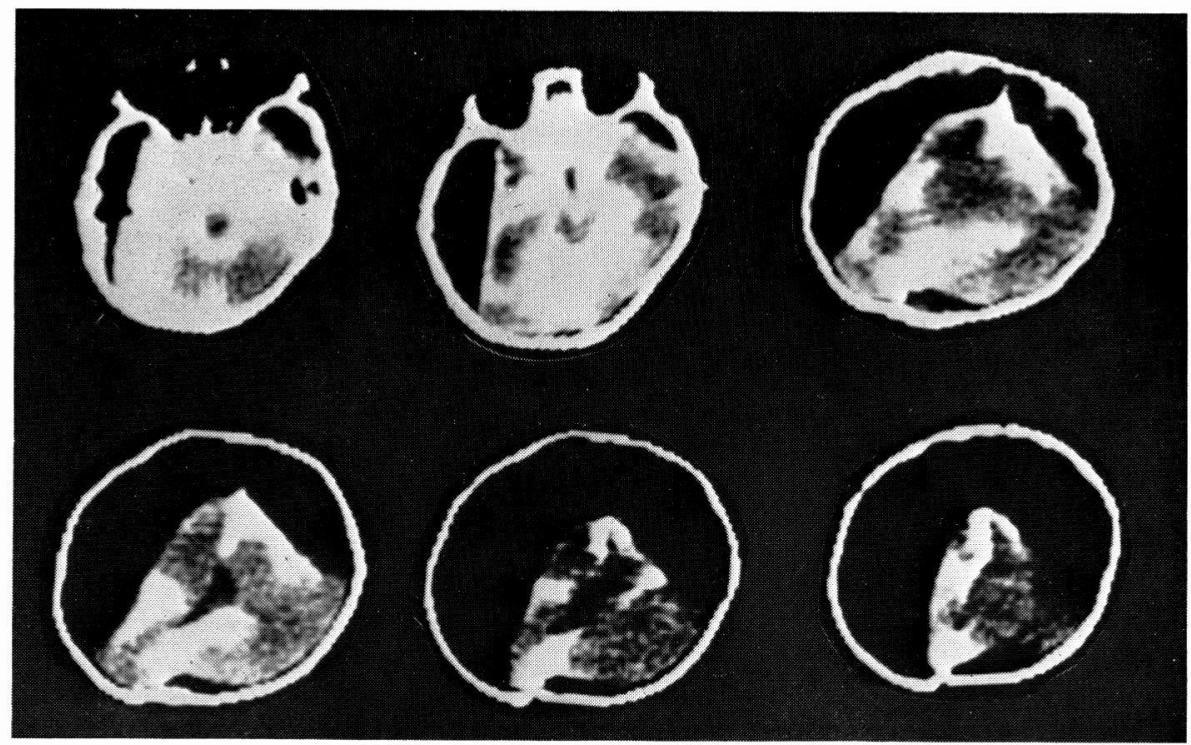

Fig. 2. The metrizamide CT cisternography is shown. The subarachnoid space in the posterior fossa was normal, but an asymmetrical formation is seen at the supratentorial sylvian fissure.

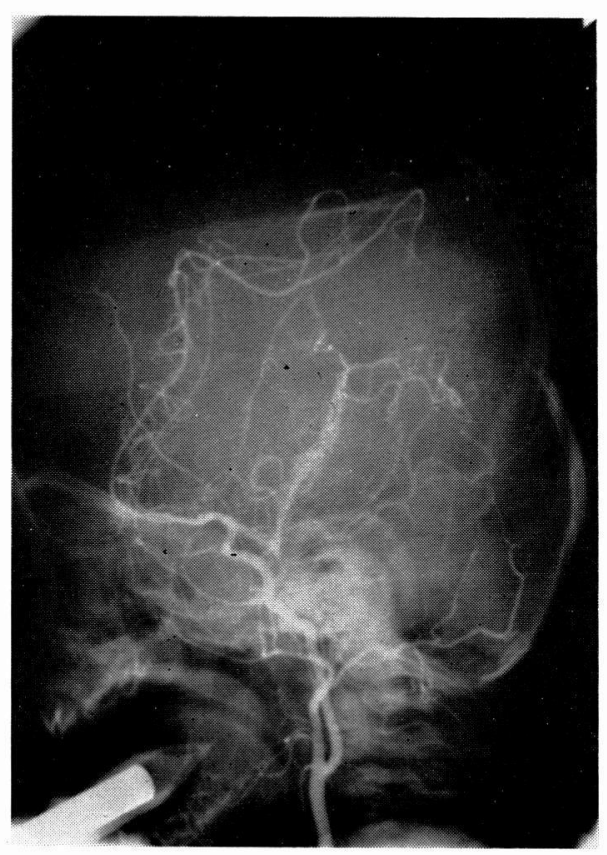

Fig. 3. The left carotid angiography (lateral view) demonstrated the anterior artery to be running along the frontal base, and the sylvian triangle is not formed.

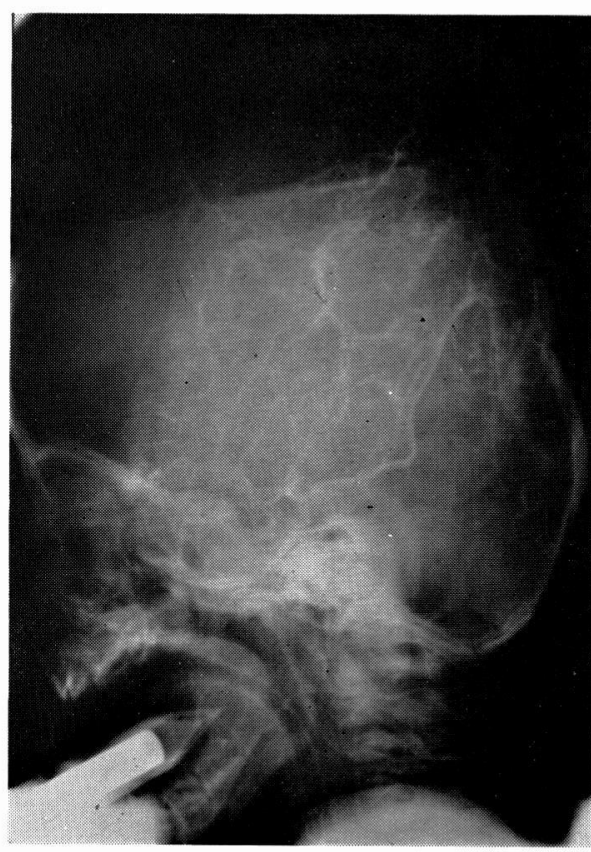

Fig. 4. Venous phase. The internal cerebral veins, inferior sagittal sinus and straight sinus are not visualized. The basal ganglia are drained by the veins which run laterally and lead to the lateral sinus. 


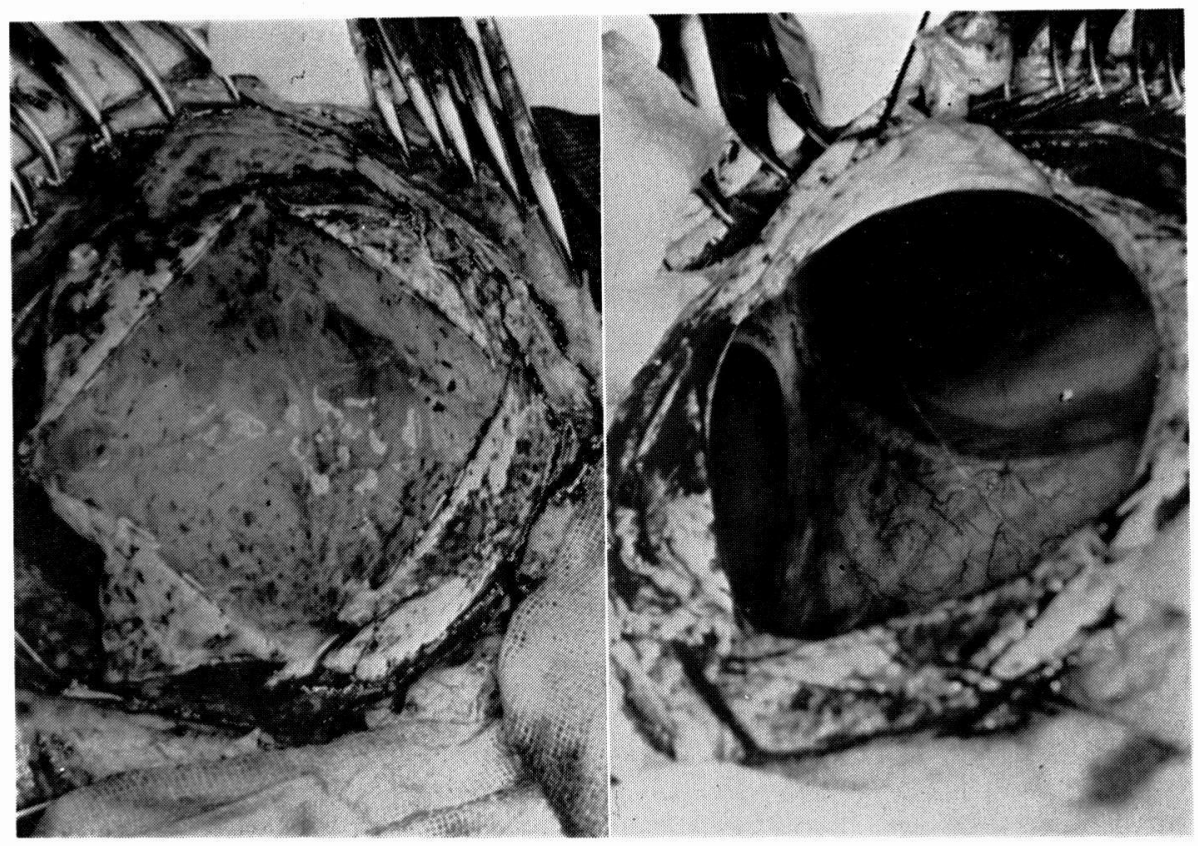

Fig. 5. Left fronto-temporal craniotomy was performed. Membrane like connective tissue was observed below the opened dura. (left) A huge fluid space is shown after removing the membrane. An incomplete gyrus is formed on the exposed brain parenchyma. A part of brain is united with the frontal base with the connecting cord. (right)

cerebral ventricle (Fig. 6).

The patient's condition was not improved and the effusion-like space was not reduced. Hypothermia at $34-35{ }^{\circ} \mathrm{C}$ and grimace was frequently observed. Sudden death due to an unknown cause occurred on October 11, 1979.

\section{Discussion}

This disease has been called by different names by different authors; Yakovlov (1959) called it arhinencephaly or holotelencephaly, whereas DeMyer and Zemen (1963) named it holoprosencephaly. This was based on the fact that the anterior cerebral vesicle existing in the embryo at 5 weeks (period of the $3 \mathrm{rd}$ up to the 5 th embryonic week) may show a disturbed differentiation. Currently this name is the accepted one.

DeMyer et al. (1963) classified the pathological severity into alobar, semilobar and lobar depending on the facial anomaly (hypotelorism, cleft palate and diastematochilia) which accompanies this disease and to the differentiation of the brain. This classification is widely accepted at present.

However, cases of holoprosencephaly with no facial anomaly and with olfactory bulb and tract which are considered essential in this disease, have been recently reported. Therefore, this anomaly is now thought to have a wide spectum of abnormalities (Nakagawa et al. 1980).

In this case report, we studied its clinical characteristics. 


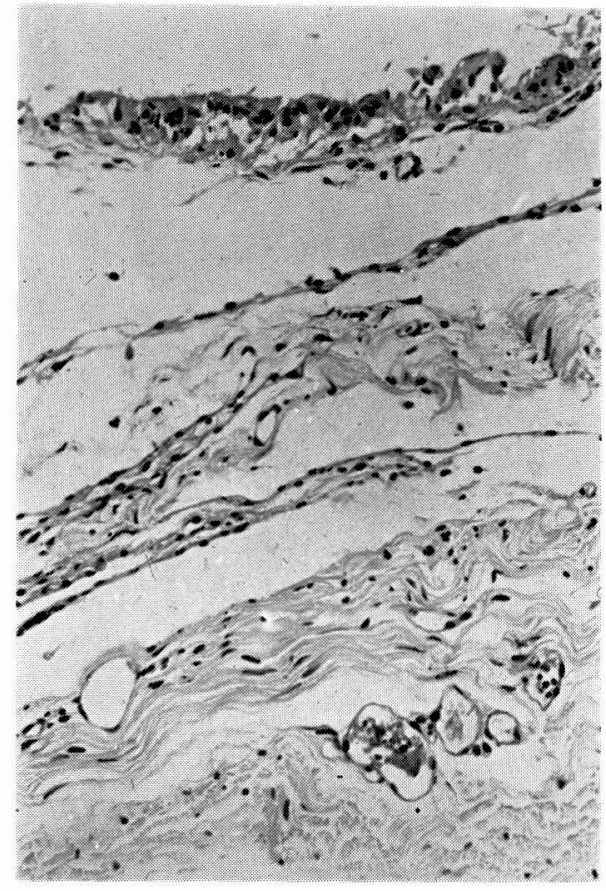

Fig. 6. Histological findings of the membrane under the dura mater demonstrated glial cells and connective tissue. The inner most layer of the membrane was covered with subependymal cells which constituted a part of the ventricles.

\section{1) Clinical symptoms}

A typical holoprosencephaly is accompanied by abnormalities of the facial cranium, such as microcephaly, ethomocephaly, or cyclopia, including hypotelorism, diastematochilia and cleft palate (Kumagai, 1971). The neurological symptoms include convulsions, which appear in many cases as in this case, as well as myoclonia, delayed mental development and poor suckling. In addition, early disappearance of the Moro's reflex or conversely, its accelerated abnormal overreaction, and apnea attack are frequently observed. Poor temprature control due to dysfunction of the hypothalamus is also noted, because of an incomplete isolation and development of the diencephalon. Sudden death, which may occur in this disease is considered as one of its characteristic findings.

\section{2) $C T$ findings}

In the alobar type, the falx cannot be identified on CT, while a part of the incomplete falx may appear in semilobar and lobar types. The cerebral ventricle is single supratentorially, while it is normal in the posterior fossa. The occupied region of the supratentorial parenchyma correspond with the dorsal sac (diencephalic cyst communicating with the cerebral ventricles) (Osaka et al. 1978). The dorsal sac was observed in the posterior and lateral cranium. In this case, metrizamide CT cisternography was also performed. The structure of the subarachnoid space in the posterior fossa was found to be normal, while the metrizamide transfer in the supratentorial system was asymmetric. Since the sylvian and longitudinal fissures were not clearly recognized, it was impossible to differentiate them from the subdural effusion.

\section{3) Angiographic findings}

The characteristic findings of holoprosencephaly were reported as follows. In the arterial phase (DeMyer et al. 1964). The proximal portion of the anterior cerebral artery runs along the frontal base, and the distal portion is tortuous. (DeMyer et al. 1964). The azygous anterior cerebral artery is observed and one of the anterior cerebral arteries is imperfectly developed. The sylvian triangle is not formed. In the venous phase, (Jin, 1979), the deep cerebral veins (straight sinus, internal cerebral vein and Galen vein) are poorly formed (Kumagai, 1971). Defect of sagittal sinus, and (Kurlander et al. 1971) a high position of the torcular Herophili. Among these findings, morphology of the deep venous system is highly important. Since the $3 \mathrm{rd}$ ventricle is retained in the early stage of the embryo, the internal cerebral vein and the straight sinus do not develop, 

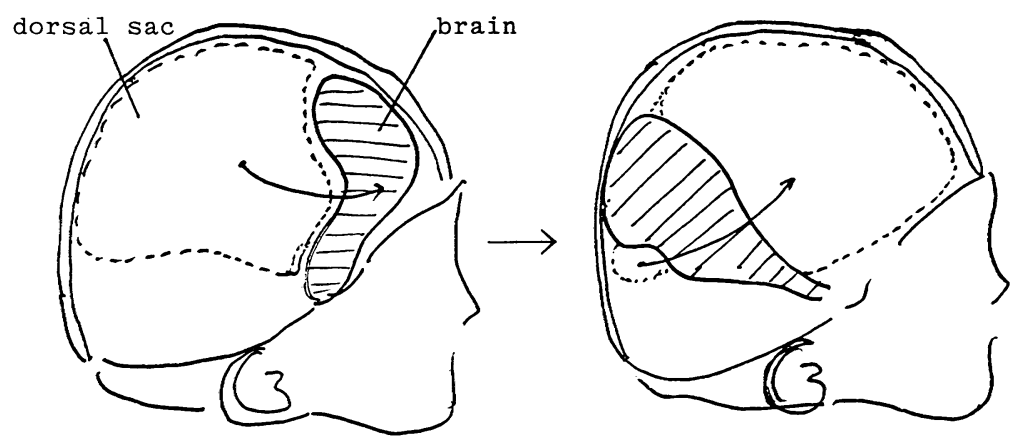

Fig. 7. Demonstration of the retroflexed holospheres.

while the emissary veins of the basal ganglia and the internal cerebrum in the embryonic state still remain and networks of the numerous abnormal veins, as observed in this case, are described (Jin, 1979, Osaka et al. 1978).

\section{4) Histological findings}

In the histological findings of the subdural membrane excised by fronto-temporal craniotomy, the wall consisted of the thin nerve cells, connective tissue and the pia mater. The inner most layer, which was in contact with the sequestrated fluid, was covered with ciliary ependymal cells. The cavity was identified to be a part of the cerebral ventricle.

The origin of the dorsal sac has been reported as velum, transversum, paraphyseal arch, diencephalic tela, choroidea, diencephalic and telecephalic roofs Actually, the tissues of the dorsal sac are mainly ependymal cells, and considered to be apart of the cerebral ventricle. In this case, the diencephalic ventricles in the process of differentiation are the most probable origin.

In some cases, the position of the dorsal sac may become abnormal as in an encephalocele ejected to the outside of the cranium (Osaka et al. 1978) and as in the retroflexional conditions of the cerebral parenchyma, reported by Kurlander (1966) and DeMyer et al. (1964), in which the dorsal sac is pushed anteriorly (Yakovlov, 1959 ((Fig. 7).

The phenomenon of retroflexion may be explained by the finding that the formation of the bridging vein, which normally drain blood to the superior sagittal sinus and protect the cerebral parenchyma from shifting, are incomplete in holoprosencephaly because the superior sagittal sinus is hypoplastic or in defective in this anomaly.

\section{Conclusion}

We report a case of 3-month-old infant with holoprosencephaly of the semilobar type manifesting as extensive subdural effusion on CT scan, but with no facial anomaly.

It was confirmed by histological examination that the subdural effusion-like space was the dorsal sac or ventricles. It seemed interesting that the dorsal sac was secondarily pushed forward by backward retroflexion of the cerebral parenchyma, as described by DeMyer and Zeman (1963).

\section{References}

DeMyer, W. and Zeman, W. (1963). Alobar holoprosencephaly (arhinencephaly) with median cleft lip and palate; clinical, electroenceph- 
alographic and nosologic considerations. Confin. Neurol. 23, 1-36.

DeMyer, W., Zeman. W. and Palmer, C. (1964). Face predicts brain diagnostic significance of median facial amomalies in holoprosencephaly (arhinencephaly). Pediatrics, 34, 256-263.

JiN, K. (1979). Angiographic and computed tomographic features of holoprosencephaly. Brain and Development. 11, 429-444.

Kumangai, K. (1971). Clinico-pathological characteristics of holoprosencephaly. Brain and Devel. 3, 460-474.

Kurlander, G. J., DeMyer, W. and Campbell, J. A. (1966). Roentgenology of holoprosencephaly (arhinencephaly). Acta. Radiol (Diagn) 5, 25-40.
Nakagawa, T., Takamatsu, H., Suematsu, K., Tsuchita, H., Mochizuki, Y. and Goto, M. (1980). Holoprosencephaly with presence of olfactory nerve - A case report and a review of literature - No to shinkei 32, 675-681.

Osaka, K. and Matsumoto, S. (1978). Holoprosencephaly in neurosurgical practice. J. Neurosurg. 48, 787-803.

PровST, F.P. (1979). The prosencephalies. pp. 77-78 Berlin, Heidelberg, New York: Springer-Verlag.

Yakovlov, P.I. (1959). Pathoarchitectomic studies of cerebral malformations III Arhinencephalies (Holotelencephalies). J. Neuropathol Exp. Neurol. 18, 22-55. 